\title{
Feedback Control Architecture of the R. sphaeroides Chemotaxis Network
}

\author{
Abdullah Hamadeh ${ }^{1,2,3}$, Elias August ${ }^{1,2}$, Mark Roberts ${ }^{1,2,4}$, Philip K. Maini ${ }^{2,5}$, \\ Judith P. Armitage ${ }^{2,4}$, Brian Ingalls ${ }^{3}$ and Antonis Papachristodoulou ${ }^{1,2}$
}

\begin{abstract}
This paper investigates the chemotaxis behavior of the bacterium $R$. sphaeroides. We review the results of a recent study comparing different possible mathematical models of this bacterium's chemotaxis decision mechanisms. It was found that only one of the aforesaid models could explain the experimental chemotactic response data. From a control theoretic perspective, we show that, compared to the other models posed, this model exhibits better and more robust chemotactic performance. This decision mechanism parallels a feedback architecture that has been used extensively to improve performance in engineered systems. We suggest that this mechanism may play a role in maintaining the chemotactic performance of this and potentially other bacteria.
\end{abstract}

\section{INTRODUCTION AND BACKGROUND}

Mathematical modeling is important for the analysis of complex pathways responsible for sensing, signalling and response mechanisms in living organisms [18]. This approach can be used to understand the dynamic behavior and robustness of biological systems, and how such properties are dependent upon the pathway's architecture [8].

One such pathway is bacterial chemotaxis, which controls bacterial motion in response to environmental stimuli, towards favorable concentrations of chemoeffectors. When certain chemical ligands are sensed, the bacterium's random walk within its medium is biased so that it moves up (or down) the ligand concentration gradient depending on the ligand's attractivity (or toxicity).

An important feature of bacterial chemotaxis is adaptation. This is a mechanism that makes the bacterium respond to changes in sensed ligands only temporarily, before restoring the bacterium's pre-stimulus random walk. The advantage of this feature is that it allows the bacterium to accurately sense a wide ligand concentration range with adequate sensitivity.

The Escherichia coli chemotaxis signalling pathway is a particular example that has been extensively studied. In [21], it was shown that the model of this pathway proposed in [1] adapts through an embedded negative feedback and integral control mechanism. With reference to the $E$. coli pathway illustrated in Fig. 1, chemical ligands bind to methyl-accepting chemotaxis protein (MCP) receptors that span the cell membrane and alter the activity of a chemotaxis protein called CheA. When attractant ligands stimulate the chemotaxis pathway by binding to MCP, there is a decrease

1: Dept. of Engineering Science, University of Oxford, Oxford, UK

2: Oxford Centre for Integrative Systems Biology, Dept. of Biochemistry, University of Oxford, Oxford, UK.

3: Dept. of Applied Mathematics, University of Waterloo, Waterloo, Ontario, Canada.

4: Dept. of Biochemistry, University of Oxford, Oxford, UK

5: Centre for Mathematical Biology, Mathematical Institute, University of Oxford, Oxford, UK.

The authors acknowledge funding from EPSRC grant EP/05708X/1 and $\mathrm{BBSRC}$ grant $\mathrm{BB} / \mathrm{F} 018479 / 1$. $\mathrm{AH}$ and $\mathrm{BI}$ acknowledge funding from the Govt. of Ontario, Ministry of Research and Innovation. MR acknowledges funding from BBSRC. PKM was partially supported by a Royal Society Wolfson Research Merit award. in the auto-phosphorylation rate of CheA. Two chemotaxis proteins, CheY and CheB, compete for the phosphoryl group from phosphorylated CheA (CheA-P). CheY-P interacts with the FliM motor protein in the motors of the $E$. coli flagella, resulting in a change in the direction of rotation of the motor. At the receptor, the protein Che R constantly methylates the $\mathrm{MCP}$ receptors, whilst CheB-P acts as a negative feedback signal that de-methylates the MCP receptors, thus reducing their activity and restoring it to the pre-stimulus level [17], [4].

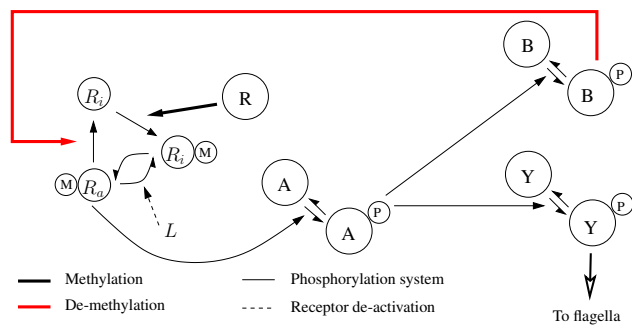

Fig. 1. The E. coli chemotaxis pathway.

Other species have chemotaxis pathways that are more complex than that of E. coli [20], [7]. The chemotaxis pathway of the bacterium Rhodobacter sphaeroides, shown in Fig. 2, contains multiple homologues of the proteins found in the E. coli pathway [7], [12], [13]. This pathway also contains two receptor clusters, one located at the cell pole and the other in the cytoplasm [19]. Ligands in the bacterium's environment regulate the activity of receptors in the two clusters. Through in vitro phosphotransfer experiments [10], [11], it is known that the CheA homologues located at the two clusters can phosphotransfer to different $\mathrm{CheY}$ and CheB homologues: at the cell pole $\mathrm{CheA}_{2}-\mathrm{P}$ phosphotransfers to $\mathrm{CheY}_{3}, \mathrm{CheY}_{4}, \mathrm{CheY}_{6}, \mathrm{CheB}_{1}$ and $\mathrm{CheB}_{2}$, while at the cytoplasm $\mathrm{CheA}_{3} \mathrm{~A}_{4}-\mathrm{P}$ phosphotransfers to $\mathrm{CheY}_{6}$ and $\mathrm{CheB}_{2}$. The CheB homologues, $\mathrm{CheB}_{1}$ and $\mathrm{CheB}_{2}$, are responsible for the adaptation mechanism in $R$. sphaeroides [12], [9] and are found throughout the cytoplasm [19]. This potentially means that the two proteins can de-methylate each of the receptor clusters [19].

As a system featuring an adaptation mechanism similar to that in $E$. coli but with multiple homologues of the $E$. coli chemotaxis proteins, it is useful to examine the $R$. sphaeroides chemotaxis pathway from a control engineering perspective [15].

In [6], an integrated model of this chemotaxis pathway was presented. It was assumed that as a dynamical system, this pathway had several parallels to that of $E$. coli, including the integral feedback controller responsible for adaptation. Additionally, the forward path of the system was based on the $R$. sphaeroides chemotaxis model developed in [14]. Under these assumptions, the study posed different possible 
feedback architectures that can arise from the dual sensory modules in $R$. sphaeroides. The relative advantages of the feedback structures were then compared from both control theoretic and biological angles.

Unlike the E. coli pathway, the different possible connectivities between the two feedback signals $\left(\mathrm{CheB}_{1}-\mathrm{P}\right.$ and $\mathrm{CheB}_{2}-\mathrm{P}$ ) and the two receptor clusters they regulate make this system a multi-input, multi-output (MIMO) feedback system. The additional degrees of freedom thus create the potential for improved feedback regulation. The additional receptor cluster not found in E. coli may be the source of disturbances, and one of the feedback architectures in [6] was shown to be superior in reducing the system's sensitivity to this and other sources of uncertainty such as protein copy number variations.

This particular connectivity has similarities to a feedback architecture commonly found in engineered control systems termed cascade control [16], which is usually employed when an output feedback controlled process can be split into two modules in cascade, with the primary module maintaining a set-point for the secondary module, which in turn controls the output of the overall process. In cases where the secondary module is susceptible to uncertainties, a cascade control design places an additional output feedback loop around the secondary module in order to reduce the sensitivity of the output to such disturbances. However, along with the reduction in sensitivity to uncertainty in the secondary module, in linear systems this additional loop causes a re-distribution of sensitivity towards uncertainty in the internal feedback path of the system.

In engineering applications, including this additional internal feedback also allows the control system designer more flexibility in increasing the external feedback gain to achieve higher bandwidth and faster system responses without losing stability. Cascade control is employed as a design principle in several engineering systems such as aircraft pitch control and in industrial heat exchangers [3], [2].

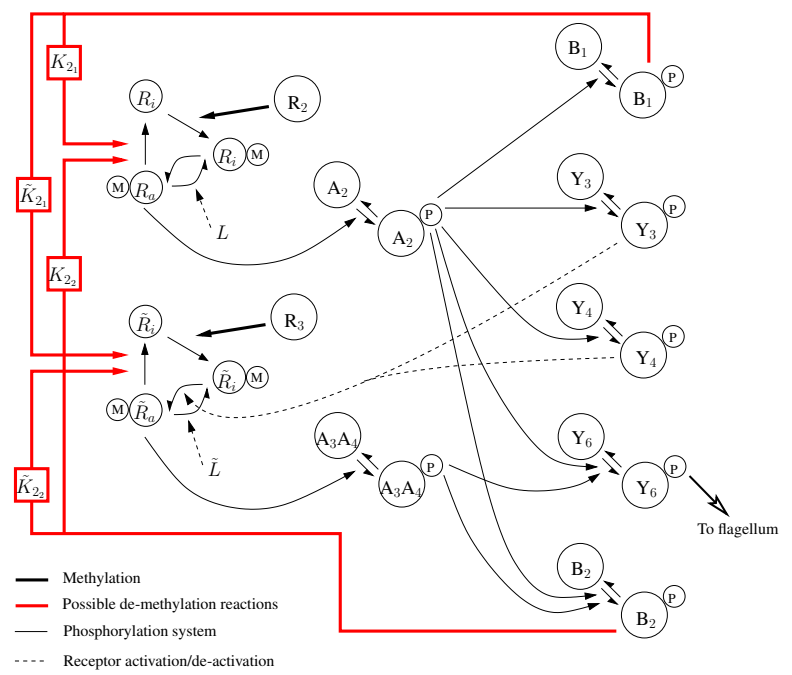

Fig. 2. The R. sphaeroides chemotaxis network, with the pathway adopted from [14].

In this paper, we begin by giving an exposition of how feedback can re-distribute sensitivity among the components of linear systems, and then apply this idea to linear systems having the cascade control architecture. We then review the main result of [6]. In light of the discussion of linear cascade control systems, we demonstrate that the (nonlinear) model suggested by [6] is structurally similar to the cascade control architecture. Furthermore, the model shares with this architecture several properties, including the sensitivity re-distribution and disturbance rejection features for which this control scheme is employed in engineering applications, suggesting that this structure may be the result of evolutionary advantages arising from increased robustness.

\section{SEnSitivity Re-Distribution}

The primary aim of using feedback in a control system is to reduce the effect of uncertainties in the system and thus maintain the desired output. Consider the block diagram in Fig. 3 which models a plant $G(s)$ with a controller $H(s)$ in negative feedback. The closed loop transfer function from input $u$ to output $y$ is given by

$$
\bar{y}(s)=\frac{G(s)}{1+G(s) H(s)} \bar{u}(s)=P(s) \bar{u}(s)
$$

where $\bar{u}(s)$ and $\bar{y}(s)$ are the Laplace transforms of $u(t)$ and $y(t)$ respectively. In the case where $G(s)$ is perturbed by uncertainties and un-modelled dynamics, the effect of an incremental change in the plant $G(s)$ on the transfer function $P(s)$ can be quantified by the sensitivity function $S_{G}(s)$ :

$$
S_{G}(s)=\frac{\frac{\partial P(s)}{P(s)}}{\frac{\partial G(s)}{G(s)}}=\frac{1}{1+G(s) H(s)}
$$

In the MIMO case, this sensitivity function takes the form $S_{G}(s)=(I+G(s) H(s))^{-1}$, where $I$ is the identity matrix.

The feedback controller $H(s)$ can reduce the magnitude of the sensitivity function of the negative feedback system $P(s)$ with respect to $G(s)$ over a range of frequencies [5]. In MIMO systems, the reduction in sensitivity can be quantified via the maximum singular value of $S_{G}(s), \bar{\sigma}\left(S_{G}\right)$.

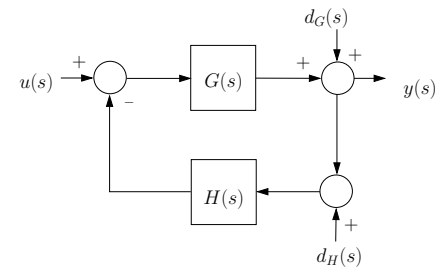

Fig. 3. A typical closed loop system.

For linear systems such as the one illustrated in Fig. 3, the sensitivity of $P(s)$ with respect to $H(s)$ is given by

$$
S_{H}(s)=\frac{\frac{\partial P(s)}{P(s)}}{\frac{\partial H(s)}{H(s)}}=-\frac{G(s) H(s)}{1+G(s) H(s)}
$$

From this relation, it is clear that for (1) there holds the sensitivity conservation constraint that, at any fixed $s \in \mathbb{C}$,

$$
S_{G}(s)-S_{H}(s)=1
$$

This relation shows that negative feedback re-distributes sensitivity between the forward and feedback paths of the system. Similarly, for a fixed $s \in \mathbb{C}$, the transfer functions from $d_{G}(s)$ to $\bar{y}(s)$ and from $d_{H}(s)$ to $\bar{y}(s)$ satisfy a conservation equation identical to (2). The transfer function $-S_{H}(s)$ (usually called the complementary sensitivity $T(s)$ ), is the transfer function from $d_{H}$ to $y$, and (2) is usually written as $S_{G}(s)+T(s)=1$. In this context, the aim is to emphasize that $S_{H}(s)$ is the sensitivity of the output with respect to the feedback $H(s)$. 


\section{CASCADE CONTROL}

The regulation scheme commonly known as cascade control is often employed in the case where there is, within a regulatory feedback loop, a sub-process that is subject to uncertainty or disturbances that directly affect the overall process output. Examples of this scheme include the control of an industrial heat exchanger [16] and aircraft pitch control [2]. With reference to Fig. 4, the method involves the use of a (secondary) feedback $H_{2}(s)$ to regulate the embedded process $G_{2}(s)$.

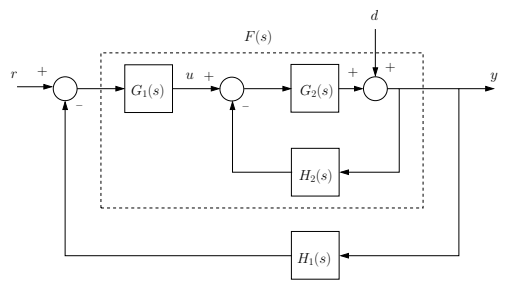

Fig. 4. Feedback system under cascade control.

Under cascade control, illustrated for the linear system in Fig. 4, the sensitivity relation (2) can be rewritten as

$$
\begin{aligned}
& \frac{F}{y} \frac{\partial y}{\partial F}-\frac{H_{1}}{y} \frac{\partial y}{\partial H_{1}}=1 \\
\Rightarrow & \frac{F}{y} \frac{\partial y}{\partial F}\left(\frac{G_{2}}{F} \frac{\partial F}{\partial G_{2}}-\frac{H_{2}}{F} \frac{\partial F}{\partial H_{2}}\right)-\frac{H_{1}}{y} \frac{\partial y}{\partial H_{1}}=1 \\
\Rightarrow & \underbrace{\frac{G_{2}}{y} \frac{\partial y}{\partial G_{2}}}_{S_{G_{2}}}-\underbrace{\frac{H_{2}}{y} \frac{\partial y}{\partial H_{2}}}_{S_{H_{2}}}-\underbrace{\frac{H_{1}}{y} \frac{\partial y}{\partial H_{1}}}_{S_{H_{1}}}=1
\end{aligned}
$$

Cascade control re-distributes sensitivity between $G_{2}, H_{2}$ and $H_{1}$. Note that, as with the trade-off between the sensitivity and complementary sensitivity, this relation leads to

$$
\left|S_{G_{2}}\right|+\left|S_{H_{2}}\right|+\left|S_{H_{1}}\right| \geq 1
$$

implying that, at any one fixed frequency, the quantities $\left|S_{G_{2}}\right|,\left|S_{H_{2}}\right|,\left|S_{H_{1}}\right|$ cannot all be made arbitrarily small. For example, if $H_{2}$ is a static gain, then, at steady state, strengthening this gain reduces $S_{G_{2}}$ and $S_{H_{1}}$ at the expense of increasing $S_{H_{2}}$. Note that $S_{G_{2}}$ is also the transfer function from $d$ to $y$ in Fig. 4, and therefore the effects of disturbances $d$ can also be reduced using the feedback $\mathrm{H}_{2}$.

In addition to the improvement of performance in terms of sensitivity reduction, cascade control enables the use of higher gain feedbacks $H_{1}(s)$ whilst maintaining stability that would otherwise be lost in the absence of the internal feedback $H_{2}(s)$. In addition, in the case where $G_{2}(s)$ is replaced by a nonlinear system $G_{2}$, a high strength static feedback replacing $H_{2}(s)$ can be used to reduce gain variations in $G_{2}$ through the linearizing effect of feedback [16], [3].

\section{A REVIEW OF PREVIOUS RESULTS}

\section{A. Modeling the R. sphaeroides chemotaxis pathway}

In this section, we briefly review the four models for the $R$. sphaeroides chemotaxis pathway presented in [6]. The pathway can be modularized into sensing, signal transduction and flagellar motor actuation subsystems, as shown in Fig. 5. Before detailing the distinguishing features of the four models, we shall describe each of these subsystems:
Sensing: The same underlying mechanisms are assumed for the polar and the cytoplasmic receptors shown in Fig. 2 (cytoplasmic cluster parameters are labelled with a tilde superscript). Some additional assumptions from the $E$. coli literature are also adopted [1]: receptors are either methylated or not, and a subset of only the methylated receptors is in an active state. Methylated receptors (active receptors) at the polar and cytoplasmic clusters are denoted by $\left[R_{m}\right],\left[\tilde{R}_{m}\right]$ $\left(\left[R_{a}\right],\left[\tilde{R}_{a}\right]\right)$ respectively. The polar and cytoplasmic clusters sense ligands of concentrations $L$ and $\tilde{L}$ respectively. Furthermore, the binding of ligands to a receptor inhibits its activity, proteins $\mathrm{CheB}_{1}-\mathrm{P}$ and $\mathrm{CheB}_{2}-\mathrm{P}$ (with concentrations $\left[B_{1_{p}}\right]$ and $\left[B_{2_{p}}\right]$ respectively) de-methylate only active receptors, and inactive receptors $\left(R_{i}, \tilde{R}_{i}\right)$ are methylated at a constant rate by $\mathrm{CheR}_{2}$ and $\mathrm{CheR}_{3}$ at the polar and cytoplasmic clusters respectively.

The parameters fitted to experimental data (see Section IVB), are $K_{1}, \tilde{K}_{1}$, the feedback matrix $\mathbf{K}_{2}=\left[\begin{array}{cc}K_{2_{1}} & K_{2_{2}} \\ \tilde{K}_{2_{1}} & \tilde{K}_{2_{2}}\end{array}\right]$ and $K_{3}, \tilde{K}_{3}$. The matrix $\mathbf{K}_{2}$ quantifies the strength of the $\mathrm{CheB}_{1}-\mathrm{P}$ and $\mathrm{CheB}_{2}-\mathrm{P}$ feedbacks to the sensing clusters (Fig. $2)$, whilst the significance of the remaining parameters is detailed in [6].

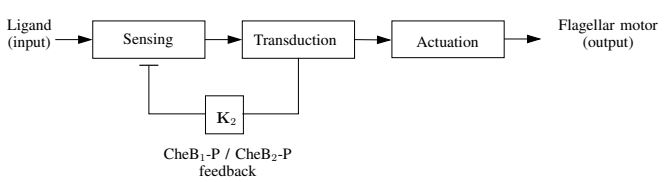

Fig. 5. Schematic of the R. sphaeroides chemotaxis pathway.

Transduction: Active polar and cytoplasmic receptors promote the auto-phosphorylation of the $\mathrm{CheA}_{2}$ and $\mathrm{CheA}_{3} \mathrm{~A}_{4}$ chemotaxis proteins, respectively. Through the phosphotransfer network (Fig. 2), phosphorylated $\mathrm{CheA}_{2}$ and $\mathrm{CheA}_{3} \mathrm{~A}_{4}$ (with concentrations $\left[A_{2_{p}}\right]$ and $\left[\left(A_{3} A_{4}\right)_{p}\right]$ ) then transfer phosphate groups to the other chemotaxis proteins [6].

Actuation: It is assumed that there exists some interaction between $\mathrm{CheY}_{6}-\mathrm{P}$ and the FliM rotor switch, which is such that $\mathrm{CheY}_{6}-\mathrm{P}$ decreases the rotation frequency of the flagellum [6].

\section{B. Experimental results and model invalidation}

In [6], four variants of the chemotaxis model above were created, each having a different feedback interconnection structure $\mathbf{K}_{2}$ between the proteins $\mathrm{CheB}_{1}-\mathrm{P} / \mathrm{CheB}_{2}-\mathrm{P}$ and the two receptor clusters. All models reproduced wild type response data and behaved as expected for the response data generated with gene deletions available at the time. The unknown parameters $K_{1}, \tilde{K}_{1}, \mathbf{K}_{2}, K_{3}, \tilde{K}_{3}$ were fitted to wild type data for each model. The four models had the following structures:

I $\quad \mathrm{CheB}_{1}-\mathrm{P}$ regulates the methylation state of the polar receptor cluster and $\mathrm{CheB}_{2}-\mathrm{P}$ of the cytoplasmic cluster only: $K_{2_{2}}=\tilde{K}_{2_{1}}=0$.

II $\mathrm{CheB}_{1}-\mathrm{P}$ regulates the methylation state of both the polar cluster and the cytoplasmic cluster while $\mathrm{CheB}_{2}$-P de-methylates only the cytoplasmic cluster receptors: $K_{2_{2}}=0$. 
$\mathrm{CheB}_{1}-\mathrm{P}$ and $\mathrm{CheB}_{2}-\mathrm{P}$ both regulate the methylation state of the polar receptor cluster and $\mathrm{CheB}_{2}-\mathrm{P}$ of the cytoplasmic receptor cluster only: $\tilde{K}_{2_{1}}=0$. IV $\mathrm{CheB}_{1}-\mathrm{P}$ and $\mathrm{CheB}_{2}-\mathrm{P}$ both regulate the methylation state of both receptor clusters.

The values of these unknown parameters were obtained in [6] for the different models by fitting them to data from tethered cell assays (for method see [14]).

After the construction of these four models, experiments were carried out to differentiate between them by finding the optimal initial conditions of the cells in the assay that maximize the difference between the outputs of the different models. When simulations of the four models were compared with experimental outputs, only Model III was able to replicate the experimental data.

\section{Numerical simulation results}

Simulations that were performed in [6] on the data-fitted Models I-IV showed how the different feedback configurations affect chemotactic performance in terms of the sensitivity of the flagellar stopping frequency in response to noise and to ligand inputs. These are summarized here.

1) Response to polar cluster noise: The bacterium's environment is typically composed of regions of high and low chemoattractant or chemorepellant concentrations. Additionally, the bacterium might sense small, fast fluctuations in the detected level of ligand due to molecular noise. To test how sensitive the chemotaxis Models I-IV are to such ligand fluctuations, each model was simulated with the sensed ligand concentration $L$ modeling a noisy signal, and the resulting rotation frequencies were then recorded. It was found that ligand level fluctuations sensed at the polar cluster of receptors resulted in larger variance of the rotation frequency in Models I, II and IV than in Model III.

2) Response to sinusoidal ligand variation: The sensitivity of the chemotaxis Models I-IV to ligand inputs was then tested in two simulations performed on each model in which the flagellar rotation frequency was recorded in response to sinusoidal variations in the ligand signals. Ligand level fluctuations sensed at the polar cluster of receptors resulted in larger changes in the rotation frequency in Models II and IV than in I and III. When the ligand concentration variations were sensed at the cytoplasmic cluster the result was a greater variation in the rotation frequency in Models I and III than in the other two models.

\section{ANALYSIS OF MODEL SENSITIVITY AND FEEDBACK}

Sections IV-B and IV-C reviewed experimental and numerical results from [6]. We next present further simulations that analyze the models' robustness properties under different feedback structures. Following these results, we use linear models with structures that represent the different connectivities of Models I-IV to analyze these structures' relative sensitivities to such disturbances.

\section{A. Model sensitivity analysis}

As the CheA proteins directly control $\mathrm{CheY}_{6_{p}}$ (which in turn controls the flagellum), their sensitivity to model uncertainty is indicative of the sensitivity of the flagellar rotation frequency. Fig. 6 shows the effects of varying the strengths of the $\mathrm{CheB}_{2}-\mathrm{P}$ feedback gains $K_{2_{2}}$ and $\tilde{K}_{2_{2}}$ to the two receptor clusters on the steady-state $\mathrm{CheA}_{3} \mathrm{~A}_{4}-\mathrm{P}$ in Model III, the model proposed in [6].

A consequence of increasing the feedback gain $K_{2_{2}}$ to the polar cluster is a decrease in the sensitivity of the steady state to the variations in $\mathrm{Che}_{3} \mathrm{~A}_{4}$ copy numbers (Fig. 6, top left) and an increase in sensitivity to variations centered around the nominal feedback gain $K_{2_{2}}$ (Fig. 6, bottom left). With regards to increasing the feedback gain $\tilde{K}_{2_{2}}$ to the cytoplasmic cluster, we observe that the sensitivity of the steady state level of $\mathrm{CheA}_{3} \mathrm{~A}_{4}-\mathrm{P}$ to copy numbers of $\mathrm{CheA}_{3} \mathrm{~A}_{4}$ is not a monotonic function of $K_{2_{2}}$ (Fig. 6, top right). We also observe an increase in sensitivity to variations centered around the nominal feedback gain $\tilde{K}_{2_{2}}$ as the nominal value of $\tilde{K}_{2_{2}}$ is increased (Fig. 6, bottom right).
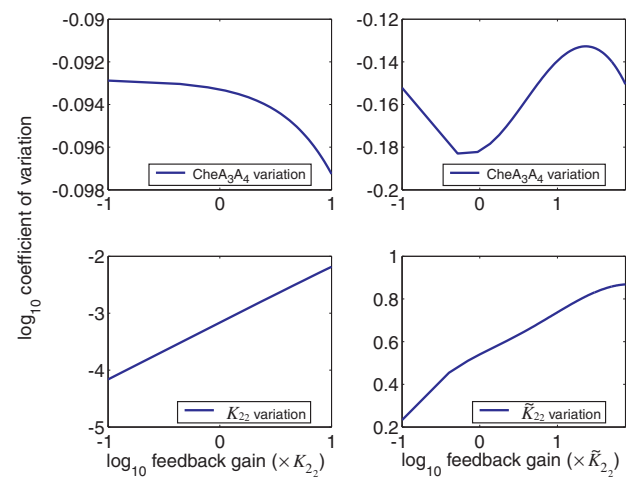

Fig. 6. Sensitivity of the steady state level of $\mathrm{CheA}_{3} \mathrm{~A}_{4}-\mathrm{P}$ to random changes in model parameters, under varying nominal feedback strengths to the polar cluster (left column) and to the cytoplasmic cluster (right column). Left: top, sensitivity to randomly varying copy numbers of $\mathrm{CheA}_{3} \mathrm{~A}_{4}$ under different feedback strengths $K_{2_{2}}$, bottom, sensitivity to random variations about nominal feedback strength $K_{2_{2}}$. Right: top, sensitivity to randomly varying copy numbers of $\mathrm{CheA}_{3} \mathrm{~A}_{4}$ under different feedback strengths $\tilde{K}_{2_{2}}$, bottom, sensitivity to random variations about nominal feedback strength $\tilde{K}_{2_{2}}$. Sensitivity is in terms of the coefficient of variation, the ratio of the standard deviation of the steady state to the nominal steady state.

The role of the internal feedback in reducing sensitivity can also be observed by first regarding the pathway as the MIMO feedback system illustrated in Fig. 7, where $G_{A}$ maps the two phosphorylated CheB proteins to phosphorylated CheA proteins, whilst $G_{B}$ maps the latter to the former. Linearizing the system, the sensitivity function

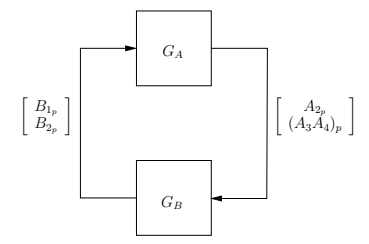

Fig. 7. MIMO representation of the R. sphaeroides chemotaxis pathway.

of the CheA protein outputs to disturbances is $S(s)=$ $\left(I+\bar{G}_{A}(s) \bar{G}_{B}(s)\right)^{-1}$, where $\bar{G}_{A}(s), \bar{G}_{B}(s)$ are the transfer functions of the linearizations of $G_{A}, G_{B}$. Fig. 8 shows the magnitude frequency response of the maximum singular values of this sensitivity function, $\bar{\sigma}(S)$, under different internal feedback strengths $\tilde{K}_{2_{2}}$ and at the operating point $L=50$, again showing that the maximum sensitivity is reduced under the strengthened internal feedback to the cytoplasmic cluster. 
The biological implications of these sensitivity observations on the performance of the chemotaxis system will be discussed in Section V-C.

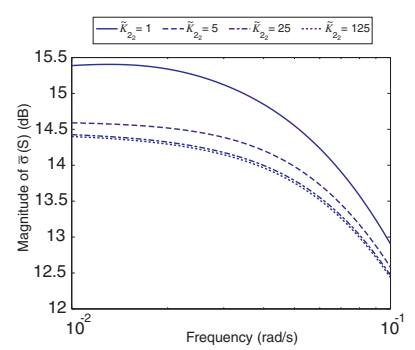

Fig. 8. Maximum singular values of the sensitivity functions of the linearized chemotaxis system at $L=50$.

\section{B. A linear chemotaxis model}

Analytic insight into how the different feedback arrangements affect the performance of the four chemotaxis models can be obtained by comparing the behavior of linear systems with structures that correspond to the different models. The block diagram in Fig. 9 shows a system composed of two modules representing the polar and cytoplasmic clusters. The outputs of the two clusters exhibit exact adaptation through integral control in response to step changes in the input ligand concentration level, as in E. coli [21]. Depending on the values of feedback gains $\widetilde{\kappa}_{2_{1}}$ and $\kappa_{2_{2}}$ (which correspond to $\tilde{K}_{2_{1}}$ and $K_{2_{2}}$ respectively in the chemotaxis models described above), the system can represent one of the four chemotaxis models:
Model I: $\quad \widetilde{\kappa}_{2_{1}}=0$ and $\kappa_{2_{2}}=0$
Model II: $\quad \tilde{\kappa}_{2_{1}}>0$ and $\kappa_{2_{2}}=0$
Model III: $\quad \tilde{\kappa}_{2_{1}}=0$ and $\kappa_{2_{2}}>0$
Model IV: $\quad \tilde{\kappa}_{2_{1}}>0$ and $\kappa_{2_{2}}>0$

The gains $a, b$ in Fig. 9 are such that $b>0, a>0$. For the examples we shall consider we set $a=1$ and $b=10$. Gains $\tilde{\kappa}_{2_{2}}>0, \kappa_{2_{1}}>0$ correspond, respectively, to $\tilde{K}_{2_{2}}$ and $K_{2}$ in the chemotaxis model. The frequency domain transfer function of the system in Fig. 9 from the ligand inputs $L$ and $\tilde{L}$ to the output $y$ is then

$$
\bar{y}(s)=\frac{\frac{b q}{s} G_{1} G_{2}}{1+\frac{a b}{s^{2}} q \kappa_{2} G_{1} G_{2}} L+\frac{G_{2}}{1+\frac{a b}{s^{2}} q \kappa_{2} G_{1} G_{2}} \tilde{L}
$$

where $G_{1}=\frac{s}{s+a \kappa_{2_{1}}}, G_{2}=\frac{s}{s+b \tilde{\kappa}_{2_{2}}}$ and $q=1-\tilde{\kappa}_{2_{1}}$.

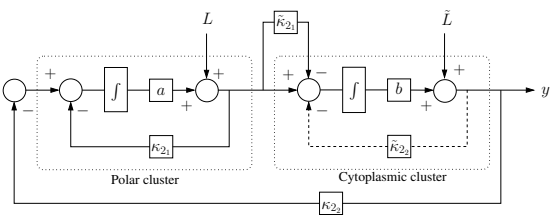

Fig. 9. Block diagram representation of a linear system structurally similar to the $R$. sphaeroides chemotaxis pathway. In this system, gain $\tilde{\kappa}_{2_{1}}$ corresponds to $\tilde{K}_{2_{1}}$ in the chemotaxis model, $\kappa_{2}$ to $K_{2_{2}}$ and $\kappa_{2_{1}}$ to $K_{2_{1}}$. Outputs of the two receptor clusters exhibit exact adaptation to step changes in ligand concentration $L, \tilde{L}$. We assume $b>0, a>0$.

Defining the return ratio of (4) as $\Lambda(s):=\frac{a b}{s^{2}} q \kappa_{2_{2}} G_{1} G_{2}$, the sensitivity of $y$ to the forward path of the system is $\Sigma_{1_{1}}(s)=$ $\frac{1}{1+\Lambda(s)}$, whilst the sensitivity of $y$ to the external feedback (with gain $\kappa_{2_{2}}$ ) is $\Sigma_{1_{2}}(s)=-\frac{\Lambda(s)}{1+\Lambda(s)}$.

Similarly, we can derive the sensitivity of $y$ to variations in the forward path of the inner cytoplasmic cluster loop of (4) as $\Sigma_{2_{1}}(s)=\frac{G_{2}(s)}{1+\Lambda(s)}$ whilst the sensitivity of $y$ to the feedback path of the inner cytoplasmic cluster loop is $\Sigma_{2_{2}}(s)=\frac{1-G_{2}(s)}{1+\Lambda(s)}$. These sensitivity functions are shown for a range of frequencies in Fig. 10.
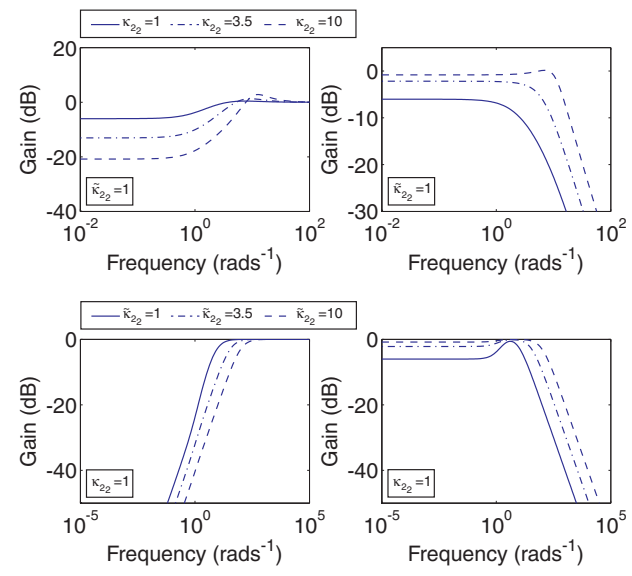

Fig. 10. Sensitivity functions of system (4) under different external $\left(\kappa_{2_{2}}\right)$ and internal $\left(\tilde{\kappa}_{2_{2}}\right)$ feedback strengths. Top left: Sensitivity $\Sigma_{1_{1}}(s)$ of $y$ to the forward path under varying feedback strength $\kappa_{2_{2}}$. Top right: Sensitivity $\Sigma_{1_{2}}(s)$ of $y$ to the feedback path under varying feedback strength $\kappa_{2_{2}}$. Bottom left: Sensitivity $\Sigma_{2_{1}}(s)$ of $y$ to the forward path under varying feedback strength $\tilde{\kappa}_{2_{2}}$. Bottom right: Sensitivity $\Sigma_{2_{2}}(s)$ of $y$ to the feedback path under varying feedback strength $\tilde{\kappa}_{2_{2}}$.

\section{The modelled and linear chemotaxis systems, compared}

The rejection of high frequency inputs at the cell pole, described in Section IV-C.1, may be advantageous in that the flagellar switching rate is then only varied when the polar cluster senses a relatively significant ligand concentration gradient that is large in spatial extent, and remains relatively unchanged when the receptors are subject to rapid fluctuations in sensed ligand due, for example, to molecular noise at the receptor.

In [6], Bode plots were used to show that the frequency response of the linear model (4) is able to qualitatively replicate the difference in gain exhibited by the two sensing clusters described in Section IV-C.2. Although the chemotaxis model assumes that the cytoplasmic cluster input depends on the sensed ligand, it is unknown what the cytoplasmic cluster senses. In addition to the possibility that this input is a function of the sensed ligand concentration, this cluster may potentially also integrate information about the metabolic state of the cell. In this case, this signalling may be important to chemotactic performance and the relatively high gain of Model III to inputs at the cytoplasmic cluster may suggest that this configuration would favor internal signals over external signals in terms of output. However, if chemotaxis is sensitive to such signals, it would be important that the cytoplasmic cluster be insensitive to variations in its biological parameters, as sensitivity to such variations would diminish the system's ability to correctly respond to inputs.

In Model III, the $\mathrm{CheB}_{2}-\mathrm{P}$ feedback loop around the cytoplasmic cluster could offer this reduction in the sensitivity of this cluster to such variations. The sensitivity function magnitude plots shown in Fig. 10 illustrate the effect of changing the values of the (internal) feedback gain $\tilde{\kappa}_{2_{2}}$ in re-distributing the sensitivity of the system (4) between the forward and feedback paths, as discussed in Section II. Increasing $\tilde{\kappa}_{2_{2}}$ reduces the sensitivity $\Sigma_{2_{1}}(s)$ of $y$ on 
the forward path of the internal feedback loop given by $\frac{b}{s}$ and increases the sensitivity $\Sigma_{2_{2}}(s)$ of $y$ with respect to variations in the internal feedback path. The feedback gain $\tilde{\kappa}_{2_{2}}$ corresponds to the gain of the $\mathrm{CheB}_{2}-\mathrm{P}$ feedback to the cytoplasmic cluster in Model III. In Fig. 6, it is shown that strengthening the $\mathrm{CheB}_{2}-\mathrm{P}$ feedback to the cytoplasmic cluster reduces the sensitivity of the steady state level of $\mathrm{CheA}_{3} \mathrm{~A}_{4}-\mathrm{P}$ to changes in the copy numbers of $\mathrm{Che}_{3} \mathrm{~A}_{4}$ and increases sensitivity in response to variations of $\tilde{K}_{2_{2}}$ away from the nominal value of $\tilde{K}_{2_{2}}$. Although the latter effect is what may be expected from the sensitivity re-distribution discussed in Section II for linear systems, in the former case, the nonlinearity of the model leads to a non-monotonic variation of sensitivity with nominal feedback strength $\tilde{K}_{2_{2}}$. Nevertheless, even for this nonlinear model, we observe that, empirically, a constraint similar to (3) holds, in that sensitivity cannot be made small at one point of the feedback path without the emergence of a corresponding increase in sensitivity at another point. If strengthening $\tilde{K}_{2_{2}}$ results in a re-distribution of sensitivity away from the parameters that strongly affect the output of the cytoplasmic cluster and towards parameters that are less likely to vary, then this may provide a mechanism by which the cluster retains a high gain to its inputs, as observed in Section IV-C.2, whilst keeping the sensitivity of its outputs to parametric variations small.

Analyzing the effects of the external feedback, we note that at steady state, increasing the feedback strength $\kappa_{2_{2}}$ decreases the sensitivity $\Sigma_{1_{1}}(s)$ of $y$ to uncertainty in the forward path and increases its sensitivity $\Sigma_{1_{2}}(s)$ to uncertainty in the feedback path. This sensitivity re-distribution effect can be observed in the chemotaxis models in Fig. 6. There, it is shown that strengthening the $\mathrm{CheB}_{2}-\mathrm{P}$ feedback gain $K_{2_{2}}$ to the polar cluster reduces the sensitivity of the steady state level of $\mathrm{Che}_{3} \mathrm{~A}_{4}-\mathrm{P}$ to changes in the copy numbers of $\mathrm{CheA}_{3} \mathrm{~A}_{4}$, whilst increasing the sensitivity of steady state $\mathrm{CheA}_{3} \mathrm{~A}_{4}-\mathrm{P}$ to changes in $K_{22}$ around its nominal value as this nominal value is increased.

\section{DISCUSSION}

The linear system (4) in the case where $\tilde{\kappa}_{2_{1}}=0$ and $\kappa_{2_{2}}>0$ has the cascade control architecture illustrated in Fig. 4. The similarities in terms of structure and behavior between this case of the linear model and Model III suggest that cascade control may be the underlying mechanism behind robust $R$. sphaeroides chemotaxis. The modularization of the chemotaxis system into the polar and cytoplasmic clusters can be seen as mirroring the modularization of the cascade controlled system in Fig. 4 into the cascade of $G_{1}$ and the feedback of $G_{2}$ with the controller $H_{2}$. The cascade control architecture enables the primary subsystem $G_{1}$ (regulated using the external feedback) to fix a set-point for the secondary system $G_{2}$ and for the feedback around the secondary system to regulate the secondary output in response to disturbances and variations in the secondary process. Model III also features both an 'internal' feedback loop nested within an 'external' one, manifested by the $\mathrm{CheB}_{2}-\mathrm{P}$ feedback that de-methylates the cytoplasmic and the polar clusters respectively. In this way, the 'external' $\mathrm{CheB}_{2}-\mathrm{P}$ feedback to the polar cluster regulates the output of the polar cluster whilst the 'internal' $\mathrm{CheB}_{2}-\mathrm{P}$ feedback to the cytoplasmic cluster regulates the output $\mathrm{CheY}_{6}-\mathrm{P}$, which in turn controls the flagellar motor.
Unlike E. coli, the $R$. sphaeroides chemotaxis pathway with cascade control feedback would consist of two feedback loops, one embedded within the other. Among the features that this delivers is a re-distribution of sensitivity to parameter variations and noise, and as long as this sensitivity is shifted to a more robust part of the feedback system, the cascade control structure can lead to a flagellar rotation frequency that is less susceptible to noise and uncertainy. Given that many chemotactic systems have multiple homologues of different important proteins [20] it would appear that using more complex feedback architectures to improve performance may be widespread in chemotaxis and in other signalling pathways, making the results of this work potentially useful for analyzing a wide set of biological systems.

\section{REFERENCES}

[1] N. Barkai and S. Leibler. Robustness in simple biochemical networks. Nature, 387:913-917, 1997.

[2] J.H. Blakelock. Automatic Control of Aircraft and Missiles, 2nd edition. Wiley- Interscience, 1991.

[3] C. Brosilow and B. Joseph. Techniques of Model-Based Control. Prentice Hall, 2002.

[4] D. Clausznitzer, O. Oleksiuk, L. Lvdok, V. Sourjik, and R.G. Endres. Chemotactic response and adaptation dynamics in Escherichia coli. PLoS Computational Biology, 6:e1000784, 2010.

[5] J. Doyle, B. Francis, and A. Tannenbaum. Feedback Control Theory. Macmillan Publishing Co., 1990.

[6] A. Hamadeh, M. Roberts, E. August, P. McSharry, P.K. Maini, J. Armitage, and A. Papachristodoulou. Feedback control architecture and the bacterial chemotaxis network. PLoS Computational Biology, 7(5):e1001130, 2011.

[7] R. Hamer, P.Y. Chen, J. Armitage, G. Reinert, and C. Deane. Deciphering chemotaxis pathways using cross species comparisons. $B M C$ Systems Biology, 4:3, 2010.

[8] L.H. Hartwell, J.J. Hopfield, S. Leibler, and A.W. Murray. From molecular to modular cell biology. Nature, 402:C47-53, 1999.

[9] A.C. Martin, G.H. Wadhams, D.S. Shah, S.L. Porter, and J.C. Mantotta et al. Cher- and cheb-dependent chemosensory adaptation system of Rhodobacter sphaeroides. Journal of Bacteriology, 183:7135-7144, 2001.

[10] S.L. Porter and J.P. Armitage. Phosphotransfer in Rhodobacter sphaeroides chemotaxis. Journal of Molecular Biology, 324:35-45, 2002.

[11] S.L. Porter and J.P. Armitage. Chemotaxis in Rhodobacter sphaeroides requires an atypical histidine protein kinase. Journal of Biological Chemistry, 279:54573-54580, 2004.

[12] S.L. Porter, G.H. Wadhams, and J.P. Armitage. Rhodobacter sphaeroides: Complexity in chemotactic signalling. Trends in Microbiology, 16:251-260, 2008.

[13] S.L. Porter, G.H. Wadhams, and J.P. Armitage. Signal processing in complex chemotaxis pathways. Nature Reviews Microbiology, 9(3):153-156, 2011.

[14] M. Roberts, E. August, A. Hamadeh, P.K. Maini, P. McSharry, J. Armitage, and A. Papachristodoulou. A model invalidation-based approach for elucidating biological signalling pathways, applied to the chemotaxis pathway in R. sphaeroides. BMC Systems Biology, 3:105, 2009.

[15] M.A. Roberts, A. Papachristodoulou, and J.P. Armitage. Adaptation and control circuits in bacterial chemotaxis. Biochemical Society Transactions, 38(5):1265-1269, 2010.

[16] D.E. Seborg, T.F. Edgar, and D.A. Mellichamp. Process Dynamics and Control. John Wiley \& Sons, 2004.

[17] T.S. Shimizu, Y. Tu, and H.C. Berg . A modular gradient-sensing network for chemotaxis in Escherichia coli revealed by responses to time-varying stimuli. Molecular Systems Biology, 6:382, 2010.

[18] J.J. Tyson, K.C. Chen, and B. Novak. Sniffers, buzzers, toggles and blinkers: dynamics of regulatory and signaling pathways in the cell. Current Opinion in Cell Biology, 15:221-231, 2003.

[19] G.H. Wadhams, A.V. Warren, A.C. Martin, and J.P. Armitage. Targeting of two signal transduction pathways to different regions of the bacterial cell. Molecular microbiology, 50:763-770, 2003.

[20] K. Wuichet and I.B. Zhulin. Origins and diversification of a complex signal transduction system in prokaryotes. Science, 3:128:ra50, 2010.

[21] T.M. Yi, Y. Huang, M.I. Simon, and J. Doyle. Robust perfect adaptation in bacterial chemotaxis through integral feedback control. Proceedings of the National Academy of Sciences, 97:4649-4653, 2000. 